\title{
Capacitive Coupling of Atomic Systems to Mesoscopic Conductors
}

\section{Citation}

Sørensen, Anders S., Caspar H. van der Wal, Lilian I. Childress, and Mikhail D. Lukin. 2004. "Capacitive Coupling of Atomic Systems to Mesoscopic Conductors." Physical Review Letters 92 (6). https://doi.org/10.1103/physrevlett.92.063601.

\section{Permanent link}

http://nrs.harvard.edu/urn-3:HUL.InstRepos:41467464

\section{Terms of Use}

This article was downloaded from Harvard University's DASH repository, and is made available under the terms and conditions applicable to Other Posted Material, as set forth at http:// nrs.harvard.edu/urn-3:HUL.InstRepos:dash.current.terms-of-use\#LAA

\section{Share Your Story}

The Harvard community has made this article openly available.

Please share how this access benefits you. Submit a story.

Accessibility 


\title{
Capacitive Coupling of Atomic Systems to Mesoscopic Conductors
}

\author{
Anders S. Sørensen, ${ }^{1,2}$ Caspar H. van der Wal, ${ }^{2}$ Lilian I. Childress, ${ }^{2}$ and Mikhail D. Lukin ${ }^{1,2}$ \\ ${ }^{1}$ ITAMP, Harvard-Smithsonian Center for Astrophysics, Cambridge, Massachusetts 02138, USA \\ ${ }^{2}$ Physics Department, Harvard University, Cambridge, Massachusetts 02138, USA
}

(Received 27 August 2003; published 13 February 2004)

\begin{abstract}
We describe a technique that enables a strong, coherent coupling between isolated neutral atoms and mesoscopic conductors. The coupling is achieved by exciting atoms trapped above the surface of a superconducting transmission line into Rydberg states with large electric dipole moments that induce voltage fluctuations in the transmission line. Using a mechanism analogous to cavity quantum electrodynamics, an atomic state can be transferred to a long-lived mode of the fluctuating voltage, atoms separated by millimeters can be entangled, or the quantum state of a solid-state device can be mapped onto atomic or photonic states.
\end{abstract}

DOI: 10.1103/PhysRevLett.92.063601

Stimulated by the emerging ideas from quantum information science, physical mechanisms that can facilitate a strong, coherent coupling of controllable quantum systems are currently being actively explored [1]. Among the leading systems being investigated are the atomic physics implementations with trapped atoms and ions $[2,3]$. These are very attractive in view of the long coherence times and the well-developed techniques for detecting and manipulating the internal states. Furthermore, the quantum states of atoms can be reversibly mapped into light, thereby providing the essential interface for quantum communication [4-7]. At the same time, solid-state systems are also being intensively investigated [8-11], and it is believed that the advanced level of microfabrication techniques could allow for the scaling of these systems to a large number of quantum bits.

This Letter describes a technique to combine the principal advantages of the atomic and solid-state systems. This is achieved by capacitively coupling a superconducting wire to neutral atoms trapped above its surface. If the atoms are temporarily excited into the Rydberg states their large electric dipole moments produce a strong interaction with the charge fluctuations in the conductor. This interaction can lead to a coupling between atomic states and a single, long-lived mode of excitation in the conductor in a manner similar to cavity quantum electrodynamics. Compared to the traditional approach of microwave cavity QED [12-14], however, transmission line resonators [15] feature a stronger confinement of the electromagnetic field, thereby significantly enhancing the relevant coupling strength.

The use of the large dipole moments of the Rydberg levels to construct quantum gates has already been proposed [16]. In free space, however, the interaction is limited to nearby atoms because the dipole-dipole interaction falls off very rapidly with the interatomic distance $(L)$ as $1 / L^{3}$. Here, the conductor acts as a waveguide for the interaction between the atoms, and the interaction strength can decrease as slow as $1 / \sqrt{L}$. Furthermore, the same approach allows one to coherently couple atomic
PACS numbers: 42.50.Pq, 03.67.Lx, 03.67.Mn, 85.25.-j

and solid-state quantum bits, since the resonator mode can interact strongly with electronic spins in quantum dots $[9,17]$ or with the charge degree of freedom of superconducting Cooper pair boxes $[11,18,19]$. This can be used to facilitate the reversible mapping of solid-state qubits to atoms, or even to optical photons with single atoms or atomic ensembles acting as mediators [4-7]. Finally, the present ideas can likely be extended to other atomic systems such as trapped ions [2]. By exploiting the dipole moment associated with the movement of an ion [20] and using small wires to connect ions trapped in separate traps, this could provide a novel route to a scalable ion trap quantum computer.

We consider the situation shown in Fig. 1. A single atom $A$ (or a small atomic ensemble) is trapped at a distance $h$ above a conducting disk of radius $R$, which in turn is connected by a thin superconducting wire to a similar disk. When atom $A$ is excited into a state with a large dipole moment, it induces a charge $q_{A}$ on the disk below it and the opposite charge $-q_{A}$ is distributed on the wire

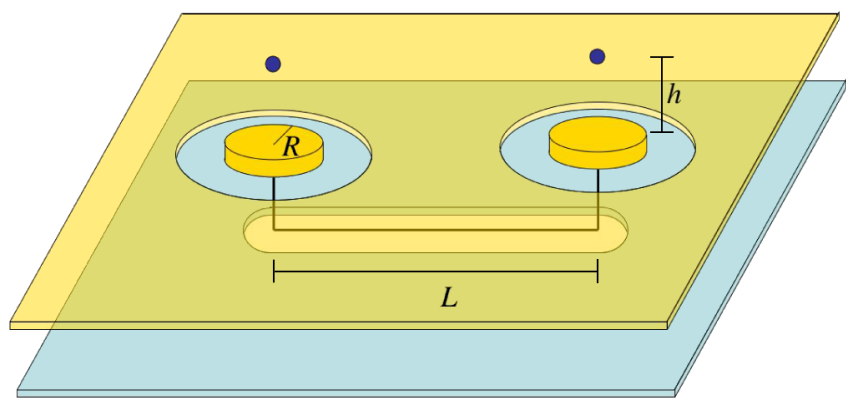

FIG. 1 (color online). Considered experimental setup (not to scale). Two atoms are located at a height $h$ above two thin normal metal disks, which are connected by a thin superconducting wire of length $L$. In the shown coplanar waveguide geometry, the wire is screened from radiative losses by a superconducting plane (lower plane). The disks and a normal metal layer (top plane) screen the wire from light used for trapping and manipulating the atoms, such that no stray light is absorbed by the superconducting wire. 
and the other disk. The second disk can be capacitively coupled to another atom $B$, or alternatively, to a solidstate quantum bit. The charge induced by atom $A$ on the disk below atom $B$ produces an electric field which interacts with the dipole moment of atom $B$, and produces an effective interaction between the two atoms. This interaction can be either electrostatic or electrodynamic in nature. In the electrostatic case, the dynamics of the atomic dipole are much slower than the resonance frequency of the wire so that the conductor adiabatically follows the atomic states and produces a static coupling between the atoms. In the electrodynamic case the resonance frequency of the atomic dipole is tuned to resonance with one of the modes of the wire producing a stronger and more flexible mechanism of interaction.

If the dynamics of the system are much slower than the resonance frequency of the wire $(\sim L / v$, where $v$ is the transmission velocity in the wire), we can neglect the inductance and the conductor is described by the Hamiltonian $H_{c}=\left(q_{A}^{2}+q_{B}^{2}\right) / 2 C_{d}+\left(-q_{A}-q_{B}\right)^{2} / 2 C_{w}$, where $C_{d}\left(C_{w}\right)$ is the capacitance of the disks (wire) and $q_{A}\left(q_{B}\right)$ is the charge on the disk below atom $A(B)$. Assuming that the interaction between the wire and the atoms is negligible, the interaction with atom $j(j=A$ or $B$ ) is given by $H_{j}=\vec{E}_{j} \cdot \vec{d}_{j}$, where $\vec{E}_{j}$ is the electric field from the charge $q_{j}$ and $\vec{d}_{j}$ is the dipole operator of atom $j$. Above the center of the disk the field is perpendicular to the disk (along the $z$ axis) and is given by $E_{z, j}=$ $q_{j} /\left(R^{2}+z^{2}\right)$ [21] so that the interaction Hamiltonian is

$$
H_{j}=\frac{q_{j} d_{z, j}}{R^{2}+h^{2}},
$$

where $d_{z, j}$ is the $z$ component of the dipole operator. Assuming that the charge on the conductor follows the atomic dipoles adiabatically, $d H / d q_{j}=0\left(H=H_{c}+\right.$ $H_{A}+H_{B}$ ), we can eliminate the charges and obtain an effective dipole-dipole interaction between the two atoms

$$
H_{\mathrm{int}}=\frac{d_{z, A} d_{z, B}}{\left(R^{2}+h^{2}\right)^{2}} \frac{C_{d}^{2}}{C_{w}+2 C_{d}} .
$$

The capacitance of a disk is $C_{d}=2 R / \pi$ [21], and to estimate the obtainable coupling we take the capacitance of the wire to be given by the expression for a coaxial cable $C_{w}=L / 2 \ln (b / a)$, where $a$ and $b$ are the inner and outer diameters of the cable, and assume $\ln (b / a) \approx 1$. In the limit $L \gg R$ with a fixed height $h$ the largest coupling is achieved with $R=h$ and we find

$$
H_{\mathrm{int}}=\frac{2}{\pi^{2}} \frac{d_{z, A} d_{z, B}}{h^{2} L} .
$$

Compared with the interaction in free space $H_{\mathrm{int}} \sim$ $d_{z, A} d_{z, B} / L^{3}$, this method provides a much stronger coupling if the atoms are close to the conductor $h \ll L$.

A stronger and more flexible coupling is possible using an electrodynamic mechanism. The finite waveguide supports a set of quantized modes of frequency $\omega_{n} \approx$ $n \pi v / L$, where $n$ is any integer. If the frequency of one of the modes in the conductor is on resonance with a transition between two Rydberg levels $\left|r_{1}\right\rangle$ and $\left|r_{2}\right\rangle$, the conductor can induce a transition between the Rydberg levels accompanied by a change of excitation in the conductor, similar to an atom emitting into a cavity. To achieve such a resonant interaction the length of the wire has to be similar to the wavelength of the Rydberg transition which is typically in the range of $\mathrm{mm}$ or $\mathrm{cm}$.

To estimate the obtainable coupling we quantize the modes in the conductor. If the wire has a charge per unit length $\lambda(x)$ and current $i(x)$, where $x$ is the coordinate along the wire, the Hamiltonian for the conductor is

$$
H_{c}=\frac{q_{A}^{2}+q_{B}^{2}}{2 C_{d}}+\int\left(\frac{\lambda(x)^{2}}{2 c}+\frac{l}{2} i(x)^{2}\right) d x,
$$

where $c$ and $l$ are the capacitance and the inductance per unit length. This Hamiltonian is diagonalized by expanding $\lambda$ and $i$ on sines and cosines and imposing the boundary condition that the potentials on the disks are the same as at the ends of the wire. Using the equations of motion $\partial \lambda / \partial t=-\partial i / \partial x$ and $\partial i / \partial t=-\left(1 / v^{2}\right) \partial \lambda / \partial x$, where $v=1 / \sqrt{l c}$, we identify the canonical momentum $p_{n}$. The Hamiltonian (4) can then be written as a sum of harmonic oscillator Hamiltonians $H_{n}=\frac{1}{2} m_{n} \omega_{n}^{2} q_{n}^{2}+$ $p_{n}^{2} / 2 m_{n}$, where $q_{n}$ is the charge on the disks associated with mode $n$, and the "mass" is $m_{n} \approx C_{w} / 2 C_{d}^{2} \omega_{n}^{2}$ (for $L \gg R$ ). Finally, we quantize the system by introducing the annihilation operators $\hat{a}_{n}=q_{n} \sqrt{m_{n} \omega_{n} / 2 \hbar}+$ $i p_{n} / \sqrt{2 m_{n} \hbar \omega_{n}}$ with commutation relations $\left[\hat{a}_{n}, \hat{a}_{n}^{\dagger}\right]=1$.

To describe the coupling between the atoms and the conductor we again use (1). If we write the dipole operator as $\hat{d}_{z}=d_{z}\left(\left|r_{1}\right\rangle\left\langle r_{2}|+| r_{2}\right\rangle\left\langle r_{1}\right|\right)$ the coupling Hamiltonian has a form familiar from cavity QED

$$
H=g\left(\left|r_{1}\right\rangle\left\langle r_{2}\left|\hat{a}^{\dagger}+\right| r_{2}\right\rangle\left\langle r_{1}\right| \hat{a}\right),
$$

where we have performed the rotating wave approximation and omitted all modes which are far from resonance with the transition. For $L \gg R$ the optimal coupling is again achieved by choosing $R=h$, and we get

$$
g=d_{z} \sqrt{\frac{2 \hbar \omega}{\pi^{2} h^{2} L}} .
$$

For a fixed transition frequency $\omega$ the coupling falls off slowly with the distance $(1 / \sqrt{L})$ and therefore permits strong coupling of atoms with a large separation. Comparing with the expression from cavity QED, $g=$ $d \sqrt{2 \pi \hbar \omega / V}$, the effective mode volume is $V=\pi^{3} h^{2} L$. The waist of a cavity mode cannot be confined to less than a wavelength and the present approach allows for a stronger coupling if $h$ is smaller than the wavelength.

The coupling in Eq. (5) is well studied and there are several proposals on how it can be exploited, e.g., for generating entanglement or quantum computation $[22,23]$. For these applications, it is essential that the quantized modes of the wire are well isolated from the 
environment. This is not the case if the wire is in free space because it would strongly radiate, and it is therefore necessary to screen the wire. Experimentally the construction of such isolated microwave resonators is being pursued and $Q$ factors of $10^{6}$ have already been achieved [15].

To get a quantitative estimate for the electrodynamic case we consider the transition from the $p$ state with principle quantum number $N$ and vanishing angular momentum along the $z$ axis to the $N-1 s$ state, and we ignore the quantum defects and the fine and hyperfine structure in the atoms. For $N \gg 1$ the matrix element for this transition is $d_{z} \approx e N^{2} a_{0} / 3 \sqrt{3}$ [24], where $e$ is the electron charge and $a_{0}$ is the Bohr radius. The coupling constant is $g \approx \hbar \omega \sqrt{2 \alpha /(3 \pi)^{3}} n \sqrt{v_{0} / v} N^{2} a_{0} / h$, where $\alpha$ is the fine structure constant, $n$ is the number of the mode which is resonant, and $v_{0}$ is the speed of light in vacuum. If we take $n=1, v \approx v_{0}, N=50$ corresponding to $\omega$ on the order of $(2 \pi) 50 \mathrm{GHz}$ or $L \sim n(3 \mathrm{~mm})$, and $h=$ $10 \mu \mathrm{m}$ we get $g \approx(2 \pi) \hbar 3 \mathrm{MHz}$. For comparison, the decoherence rate associated with Rydberg excitations can be in the range of $\mathrm{kHz}$ [13], and the decoherence rate for an excitation in the conductor is $(2 \pi) 50 \mathrm{kHz}$ for $Q \sim 10^{6}$.

We next consider several practical aspects associated with the present technique. A major concern is the van der Waals forces on the atoms from the nearby conductor [25]. Ideally one would like to trap the Rydberg atoms to ensure that the motional state remains decoupled from the evolution of the internal states. Such trapping of Rydberg atoms has not yet been demonstrated, but even without trapping the Rydberg levels it is still possible to avoid a significant deterioration due to the motion of the atoms. If we assume that the atoms are initially in their electronic ground state, which can be trapped, e.g., by optical dipole traps or microfabricated magnetic traps $[26,27]$, one can quickly excite the atoms into the Rydberg levels with a resonant pulse, let them interact with the wire, and quickly deexcite them after the interaction. A simple estimate of the force can be found from the energy of a dipole above a plane $\Delta E=-\left\langle 2 \hat{d}_{z}^{2}+\hat{d}_{\rho}^{2}\right\rangle / 16 h^{3}$, where $\hat{d}_{\rho}$ is the component of the dipole operator perpendicular to the $z$ axis. For the numerical example considered above this gives energy shifts $\Delta E \sim-(2 \pi) \hbar 1 \mathrm{MHz}$. Adding the resonant contribution we find that the maximal force experienced by an atom is $F \leq(3 \Delta E-g) / h$. If we assume that the atoms are initially cooled to the motional ground state of a trap with a trapping frequency $\nu$, and that they are temporarily transferred to an untrapped Rydberg state for a time $t$, the probability to be excited out of the ground state is $P \approx F^{2} t^{2} / 2 \hbar M \nu+\nu^{2} t^{2} / 8$, where $M$ is the mass of the atom. For a $\mathrm{Rb}$ atom with $\nu=(2 \pi) 50 \mathrm{kHz}$ this gives $P \approx 10^{-3}$ for an interaction time $t=\hbar \pi / g$.

In addition to the van der Waals forces, the Rydberg atom will also be influenced by fluctuating potentials from the surface. In current experiments with small superconducting quantum devices, the coherence times are limited by such charge fluctuations in the solid-state environment [19]. These effects are not yet fully understood and the experimental investigation is complicated by the lack of probes which do not share the same environment. The Rydberg atoms could be used as a sensitive probe of these fluctuations that is controllably separated from the device itself. If the atoms are excited into a state with a large angular momentum where the Stark shift is linear, a single electron charge added to an island of $R=$ $10 \mu \mathrm{m}$ can shift the frequency of an $N=50$ Rydberg atom located $10 \mu \mathrm{m}$ away by $340 \mathrm{MHz}$. These effects are, however, much less important for the electrodynamic coupling since the nondegenerate $s$ and $p$ states experience a much weaker quadratic Stark shift. Noise in the solid states environment will cause voltage fluctuations of the wire of order $\left\langle V^{2}\right\rangle \sim k_{b} T / C_{w}$, where $T$ is the temperature, and we estimate that these fluctuations play a negligible role because of the large capacitance of the wire. In addition the so-called patch potentials will influence the atoms [28]. As a specific example we estimate that the measured patch potentials in Ref. [25] would cause an energy shift of $\sim 7 \mathrm{MHz}$ at $h=10 \mu \mathrm{m}$. This shift however can be compensated as long as it does not change in time, e.g., due to the movement of impurities on the surface. At low temperatures such large-scale motions freeze out, thus reducing the patch field fluctuations to an acceptable level for the electrodynamic experiments.

One of the main challenges for the experimental realization involves combining strong optical control fields (needed for trapping and manipulation of atoms) with the mesoscopic superconductors. If light is incident on the superconductor the absorption of photons can break up a large number of Cooper pairs. Even when heating effects are minimal, this changes the inductance of the superconducting resonator [15] and causes decoherence of the oscillating mode. This problem can be avoided by a design in which the disks at the ends of the transmission line are made of a normal metal. The rest of the transmission line, the superconducting part, can be shielded by adding a metal layer as shown in Fig. 1. Using here a normal metalsuperconductor sandwich combines low Ohmic losses for the bottom side with good cooling of the screening layer on the top side. Note that since the voltage on the disk is determined by the total capacitance (which is mainly due to the long wire) this metal layer does not affect the coupling strength significantly. Because the current in the conductor makes a standing wave with a node at the end, the finite conductivity of the disks does not affect the resonator significantly. To estimate the obtainable $Q$ we replace the entire conductor in Fig. 1 with a wire of length $L=3 \mathrm{~mm}$ where the last $10 \mu \mathrm{m}$ in both ends are made of gold. With a conducting area of $\sim 1 \mu \mathrm{m}^{2}$ the low resistance of gold (for a thin layer at low temperature) makes a negligible contribution $\left(Q \geqslant 10^{8}\right)$. Another concern would be the resistance in the contacts between the metals, but even with $\sim 0.1 \Omega$ contact resistance $Q \sim 10^{7}$. In practice radiative losses and loss in the dielectric limit the 
ultimate value of the $Q$ factor. Because of the pillars rising above the plane, the structure we propose in Fig. 1 differs slightly from the structures used for the experiments [15]. We estimate the radiative losses from these pillars to limit $Q$ to roughly $Q \lessgtr(L / H)^{4}$, where $H$ is the height of the pillars, and hence this modification has a negligible contribution for $H \lesssim 30 \mu \mathrm{m}$. In addition the radiative loss can be suppressed by surrounding the setup with a Faraday cage, with the top plane in Fig. 1 as one of the walls.

The above geometry allows one to reduce heating effects from absorbing stray light to an acceptable level. With milliwatts of power in the beams focused on the atoms, stray light power at a level of nanowatts can be expected to be absorbed by the reflecting normal metal disk. Even at an operating temperature of $100 \mathrm{mK}$, the $10 \mu \mathrm{m}$ disks will easily have sufficient cooling power to the dielectric and the nearby normal metal plane. Quasiparticle diffusion into the superconducting wire can be minimized using a material with fast quasiparticle relaxation and a short coherence length (such as $\mathrm{NbN}$ ), while increasing the dielectric spacing will further reduce the sensitivity of the inductance to the Cooper pair density (desirable in contrast to [15]). Although subKelvin regimes are desirable for low thermal occupation of the oscillator modes, operation at temperatures as high as a few Kelvin might be feasible by using atoms to cool the relevant modes [14] or by involving techniques analogous to Refs. [12,29].

To summarize, we have presented a feasible method to coherently couple atoms to a mesoscopic conductor with a coupling strength significantly exceeding the decoherence rates. It allows one to combine solid-state microfabrication techniques with the excellent coherence properties and controllability of atomic systems. Using the proposed technique atoms separated by millimeters can be entangled and one can imagine using this to create scalable architectures for quantum computation where atoms are connected by wires. Also, the technique can be used to couple solid-state quantum bits to individual atoms or small atomic ensembles. The former can thereby be reversibly coupled to light. A different application of the ideas presented here could be the nondestructive measurement of the presence of a Rydberg atom by measuring the induced charge with a single electron transistor [30], or alternatively Rydberg atoms could be used to probe the noise in solid-state systems.

The full realization of the present proposal requires a number of technological advances toward trapping and manipulation of highly excited atoms in the vicinity of surfaces. Various elements of the proposed technique can, however, be probed with existing technology. In particular, the basic mechanism of the capacitive coupling between atoms and transmission lines can be probed using a beam of highly excited atoms directed over the conductor using the techniques demonstrated in Refs. [25,31].
We are grateful to M. Brune, S. Haroche, J.-M. Raimond, G. Reithel, D. Vrinceanu, J. Zmuidzinas, and P. Zoller for useful discussions. This work was supported by the NSF through the Career program and its grant to ITAMP, ARO, the Sloan and the Packard Foundations, the Danish Natural Science Research Council, and the Hertz Foundation.

Note added. - After submission of this work a related preprint appeared [32] which describes an interface between a trapped ion and a solid-state quantum computer using the static version of the capacitive coupling.

[1] Special issue on implementations of quantum computers [Forschr. Phys. 48, 769-1138 (2000)].

[2] C. A. Sackett et al., Nature (London) 404, 256 (2000).

[3] O. Mandel et al., Phys. Rev. Lett. 91, 010407 (2003).

[4] A. Kuhn, M. Hennrich, and G. Rempe, Phys. Rev. Lett. 89, 067901 (2002).

[5] J. McKeever et al., Phys. Rev. Lett. 90, 133602 (2003).

[6] A. Kuzmich et al., Nature (London) 423, 731 (2003).

[7] C. H. van der Wal et al., Science 301, 196 (2003).

[8] D. Vion et al., Science 296, 886 (2002)

[9] D. Loss and D. P. DiVincenzo, Phys. Rev. A 57, 120 (1998).

[10] I. Chiorescu et al., Science 299, 1869 (2003).

[11] Y. Makhlin, G. Schön, and A. Shnirman, Nature (London) 398, 305 (1999).

[12] S. Osnaghi et al., Phys. Rev. Lett. 87, 037902 (2001).

[13] M. Weidinger et al., Phys. Rev. Lett. 82, 3795 (1999).

[14] A Rauschenbeutel et al., Science 288, 2024 (2000).

[15] P. K. Day et al., Nature (London) 425, 817 (2003); B. A. Mazin et al., Proc. SPIE-Int. Soc. Opt. Eng. 4849, 283 (2002).

[16] D. Jaksch et al., Phys. Rev. Lett. 85, 2208 (2000).

[17] L. I. Childress, A. S. Sørensen, and M. D. Lukin, quantph/0309106.

[18] S. M. Girvin et al., cond-mat/0310670.

[19] Y. Nakamura et al., Phys. Rev. Lett. 88, 047901 (2002).

[20] J. I. Cirac and P. Zoller, Nature (London) 404, 579 (2000).

[21] J. D. Jackson, Classical Electrodynamics (Wiley, New York, 1962).

[22] T. Pellizzari et al., Phys. Rev. Lett. 75, 3788 (1995).

[23] P. Domokos et al., Phys. Rev. A 52, 3554 (1995).

[24] H. A. Bethe and E. E. Salpeter, Quantum Mechanics of One- and Two-Electron Atoms (Plenum, New York, 1977).

[25] V. Sandoghdar et al., Phys. Rev. A 53, 1919 (1996).

[26] R. Folman et al., Phys. Rev. Lett. 84, 4749 (2000).

[27] J. Reichel, W. Hänsel, and T.W. Hänsch, Phys. Rev. Lett. 83, 3398 (1999).

[28] Q. A. Turchette et al., Phys. Rev. A 61, 063418 (2000).

[29] A. Sørensen and K. Mølmer, Phys. Rev. Lett. 82, 1971 (1999).

[30] M. H. Devoret and R. J. Schoelkopf, Nature (London) 406, 1039 (2000).

[31] S. Dapore-Schwartz, N. G. Woodard, and G. P. Lafyatis, Phys. Rev. B 55, 5655 (1997).

[32] L. Tian et al., quant-ph/0310083. 